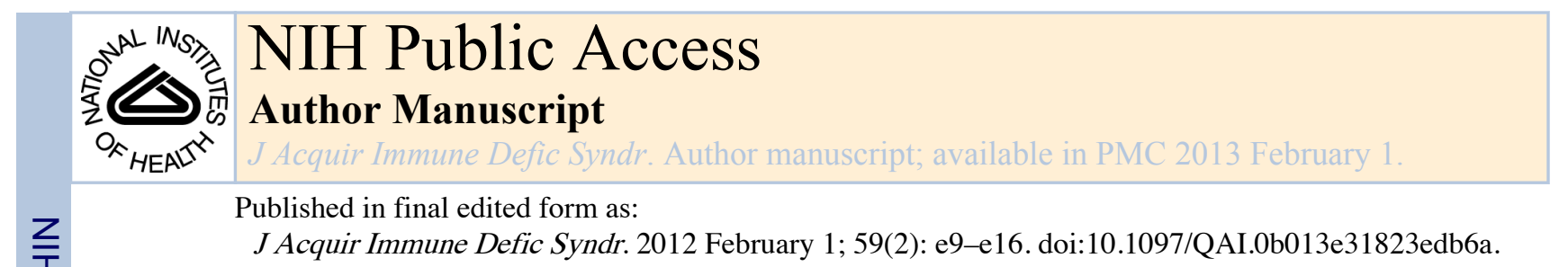

\title{
Outcomes of antiretroviral treatment programs in rural Southern Africa
}

\author{
Gilles Wandeler, MD, MSc ${ }^{1,2}$, Olivia Keiser, PhD $^{1}$, Karolin Pfeiffer, MD MPH ${ }^{3}$, Sabrina \\ Pestilli, MD, MPH ${ }^{3}$, Christiane Fritz, MD $^{3}$, Niklaus D. Labhardt, MD, MIH $^{3}$, Franzisco \\ Mbofana, MD, MPH ${ }^{4}$, Robert Mudyiradima, MD, MPH ${ }^{5}$, Jan Emmel, $\mathrm{MPH}^{3}$, Matthias Egger, \\ MD, MSc ${ }^{1}$, and Jochen Ehmer, MD, MA ${ }^{3}$ for the SolidarMed ART program and leDEA- \\ Southern Africa
}

${ }^{1}$ Division of International and Environmental Health, Institute of Social and Preventive Medicine (ISPM), University of Bern, Bern, Switzerland ${ }^{2}$ Infectious Diseases Clinic, Bern University Hospital, Bern, Switzerland ${ }^{3}$ SolidarMed, Lucerne, Switzerland ${ }^{4}$ Ministry of Health, Mozambique ${ }^{5}$ Ministry of Health, Zimbabwe

\begin{abstract}
Background-Data on outcomes of antiretroviral treatment (ART) programs in rural subSaharan African are scarce. We describe early losses and long-term outcomes in six rural programs in Southern Africa with limited access to viral load monitoring and second-line ART.

Methods-Patients aged $\geq 16$ years starting ART in two programs each in Zimbabwe, Mozambique and Lesotho were included. We evaluated risk factors for no follow-up after starting ART and mortality and loss to follow-up (LTFU) over 3 years of ART, using logistic regression and competing risk models. Odds ratios and sub-distribution hazard ratios, adjusted for gender, age category, CD4 category and WHO stage at start of ART are reported.
\end{abstract}

Results-Among 7,725 patients, 449 (5.8\%) did not return after initiation of ART. Over 9,575 person-years, $698(9.6 \%)$ of those with at least one follow-up visit died and 1,319 (18.1\%) were LTFU. At 3 years the cumulative incidence of death and LTFU were $12.5 \%(11.5-13.5 \%)$ and $25.4 \%$ (24.0-26.9\%), respectively, with important differences between countries: in Zimbabwe $75.1 \%(72.8-77.3 \%)$ were alive and on ART at 3 years compared to $55.4 \%(52.8-58.0 \%)$ in Lesotho and 51.6\% (48.0-55.2\%) in Mozambique. In all settings young age and male gender predicted LTFU, whereas advanced clinical stage and low baseline CD4 counts predicted death.

Conclusions-In African ART programs with limited access to second-line treatment, mortality and LTFU are high in the first 3 years of ART. Low retention in care is a major threat to the sustainability of ART delivery in Southern Africa, particularly in rural sites.

\section{Keywords}

Antiretroviral treatment; Cohort study; rural Southern Africa; retention in care

\footnotetext{
Corresponding author: Gilles Wandeler, MD, MSc, Institute of Social and Preventive Medicine (ISPM), Finkenhubelweg 11, CH-3012 Bern, Switzerland, Phone: +41 3163138 67, Fax: +41 3163135 20, gwandeler@ispm.unibe.ch. AUTHOR CONTRIBUTIONS

GW, OK and ME designed the study, conducted the statistical analyses and drafted the paper. KP, SP, CF, NL, FM, SC and JE contributed data, critically revised the paper and approved its final version. JE contributed to the design and implementation of the SolidarMed ART program. All authors approved the final version of the paper.

Conflicts of Interest and Sources of Funding: Dr. Ehmer, Dr. Pfeiffer \& Dr. Pestilli have received funding for travel and other expenses related to this study from SolidarMed. No other conflicts of interest or sources of funding exist.
} 


\section{INTRODUCTION}

The World Health Organization (WHO) estimates that over 5 million HIV-1 infected people were receiving antiretroviral therapy (ART) in low- and middle-income countries by the end of $2009^{1}$. However, high mortality and loss of patients to follow-up in the context of the rapid scale-up of ART and weak health systems challenge clinical and programmatic outcomes in low-income settings in Africa. ${ }^{2,3}$ In recent years an increasing body of evidence, including two systematic reviews, has shown low retention in care of patients starting ART in sub-Saharan Africa. ${ }^{4-7}$ Early losses after the initiation of ART have been recognized as important barriers to the success of ART programs in resource-constrained settings. ${ }^{8,9}$ According to a recent report based on aggregate data from national programs, retention in care in countries in sub-Saharan Africa is estimated to be as low as $75.2 \%$ at 12 months, possibly due to high early mortality. ${ }^{10}$

Data on short-term and long-term clinical and programmatic outcomes in rural sub-Saharan Africa, especially in settings with limited diagnostic possibilities, access to viral load testing and second-line ART, are scarce. Due to many structural factors influencing the scale-up of ART, the distribution of services may be inequitable between rural and urban African settings. ${ }^{11}$ For instance, the impact of the chronic shortage of health care workers has been most devastating in rural settings. ${ }^{12}$

Mozambique, Zimbabwe and Lesotho are three countries with a high prevalence of HIV infection where ART delivery has been scaled up in the last few years. Despite the substantial increase in patients starting ART, only $30 \%, 34 \%$ and $48 \%$ of the HIV-infected population in need of treatment in Mozambique, Zimbabwe and Lesotho, respectively, were estimated to have access to ART by $2009 .{ }^{1}$ In this context, SolidarMed, a Swiss NonGovernmental Organization (NGO), established the SolidarMed ART (SMART) program in $2005,{ }^{13}$ with a focus on supporting the delivery of ART and health care in rural settings in sub-Saharan Africa. We examined the importance of no follow-up after initiation of ART as well as mortality and loss to follow-up (LTFU) over three years of ART in the SMART programs in Zimbabwe, Mozambique and Lesotho.

\section{METHODS}

\section{SolidarMed AntiRetroviral Treatment (SMART) program}

The SMART program was launched in rural Zimbabwe, Mozambique and Lesotho in 2005 to support the national ART programs by introducing voluntary testing and counseling (VCT) services, antiretroviral drugs for the prevention of mother-to-child transmission (PMTCT) of HIV, and by providing ART and treatments of opportunistic diseases. While national authorities and health systems continued to manage services, SolidarMed facilitated implementation, bridged gaps in funding and supported capacity building and monitoring. Subsequently the program was expanded to 9 district health facilities and over 40 affiliated peripheral health centers in Lesotho (3 districts), Zimbabwe (2 districts), Mozambique (2 districts) and Tanzania (2 districts).

All ART programs included in this study are rural. Sixty percent of patients in Lesotho and $90 \%$ of those in Mozambique and Zimbabwe were subsistence farmers and $80 \%$ were illiterate. Every ART program has a well defined catchment area, which includes between 55,000 people in Lesotho and 240,000 in Mozambique. Programs are nurse-led, with only 2 medical doctors in Mozambique, 4 in Lesotho and 5 in Zimbabwe, and there is usually only one functioning ambulance per area. Finally, most patients travel between 1 and 3 hours to 
the clinic and distances to the next referral clinic can be up to 240 kilometers, depending on the area.

The programs in Zimbabwe, Mozambique and Lesotho are part of the International epidemiologic Databases to Evaluate AIDS in Southern Africa (IeDEA-SA), a collaboration of ART programs from seven countries in the region. The IeDEA networks in sub-Saharan Africa have been described in detail elsewhere. ${ }^{14}$ In the present study we analyzed data from the SMART programs in Zimbabwe, Mozambique and Lesotho.

\section{Data collection}

SolidarMed supports the monitoring of ART programs through the provision and maintenance of a purpose-built clinical database. Data entry clerks are trained by senior local data managers. Data are prospectively recorded on standardized national patient forms by clinical nurses and entered by data clerks into the database, which is the same at all sites. Data collection starts on the day ART is initiated and continues at each visit to the clinic. Information on demographic characteristics such as date of birth, marital status, occupation, residency as well as past medical history, including year of diagnosis of HIV infection and prior CD4 counts is recorded on the first day. Clinical parameters, including height, weight, opportunistic infections and WHO stage and laboratory parameters are recorded at baseline and during follow-up. Treatment adherence is monitored by two means: pill counts and adherence to medical appointments.

Local data supervisors regularly perform random quality checks on site, comparing entered data with paper forms. Twice a year, an additional quality-check is performed with the help of staff at SolidarMed head-quarters in Lucerne, Switzerland and the Institute of Social and Preventive Medicine (ISPM) at the University of Bern, Switzerland. Personal identifiers are removed before transfer of the data for statistical analysis. The present analysis includes all data available up to the end of June 2010.

\section{Counseling, follow-up and tracing of patients}

Three individual or group adherence counseling sessions are normally held before the initiation of ART and further adherence counseling takes place during ART, if required. Most patients are seen by nurses and follow-up visits are scheduled according to national algorithms, generally every month. All new clinical events, such as pregnancy and opportunistic infections are recorded whenever patients seek medical care. CD4 counts are determined every 3 to 6 months, depending on the clinic. Further laboratory examinations include hemoglobin, total white cell count, total lymphocyte count, platelets, alanine transaminase (ALAT) and creatinine (only in Lesotho). All changes to the antiretroviral treatment regimens, serious adverse effects and the prescription of prophylactic treatments are recorded. There are no death registries in the countries involved and ascertainment of deaths occurring outside health facilities therefore relies on reports from outreach workers or direct information by families, friends or local authorities. Whenever patients have missed their last appointment at the treatment site, they are traced through home visits by nurses, volunteers, lay counselors or community associations.

\section{Inclusion criteria and outcomes}

All patients aged 16 years or older with complete data on sex and date of birth who started ART consisting of at least 3 drugs between January 2005 and June 2010 were included in the analysis. We examined three outcomes: no follow-up after starting ART, LTFU and death. LTFU was defined as not returning to the facility for 6 months or longer. Patients who started ART within the last 6 months before the closing date of this analysis were therefore excluded from this study. Follow-up of patients transferred to another clinic was 
censored at the date of their last visit. Retention in care was defined as alive on ART and in active follow-up over the study period.

\section{Statistical analyses}

We identified risk factors for no follow-up after starting ART using logistic regression. Variables at the start of ART included in the model were sex, age (16-29, 30-39, 40 years and over), CD4 cell count (0-49, 50-99, 100-199, 200 cells/ $\mu \mathrm{L}$ and over), WHO stage (I/II or III/IV), calendar year (before 2008, 2008 and after 2008), country and initial treatment regimen (Nevirapine- or Efavirenz-based).

We used logistic regression to identify factors associated with not returning to the clinic after initiation of ART, and competing risk models to analyzed mortality and LTFU. Death is a competing risk of LTFU: patients who die can no longer become lost to follow-up. Similarly, deaths in patients who are lost to follow-up are no longer recorded in the database. Standard time-to-event analyses, for example Kaplan-Meier curves or Cox regression, ignore the competing risks of death and LTFU and may produce biased results. We therefore plotted stacked competing risk cumulative incidence curves of LTFU and mortality up to three years after starting ART. ${ }^{15}$ Furthermore, we used the competing risk sub-distribution models described by Fine and Gray ${ }^{16}$ to examine risk factors for LTFU or death. All statistical analyses were performed using Stata (version 11, Stata Corporation, College Station, TX, USA).

\section{Ethical approval}

We obtained ethical approval for the collection and transfer of data from the Comité Nacional de Bioética para a Saude, Republica de Moçambique, the Medical Research Council of Zimbabwe and the Ministry of Health and Social Welfare of Lesotho for the SMART programs in Mozambique, Zimbabwe and Lesotho, respectively. In the case of Lesotho, the ethical clearance was issued to SolidarMed and its main partner, the Christian Health Association of Lesotho (CHAL). In Mozambique, the ethics committee required individual informed consent, whereas informed consent was not required in Zimbabwe or Lesotho.

\section{RESULTS}

\section{Characteristics of clinics and patients}

The six ART programs differed in terms of the number of nurses, medical officers and doctors seeing patients, travel times of patients, and first-line regimens used (Table 1). Staffing was lowest in Mozambique, where only around 20 nurses and 1 or 2 medical doctors were available per 100,000 population. The mean travel time to the clinic ranged from 1 hour in Zaka, Zimbabwe, to 3 hours in Ancuabe, Mozambique. All sites except Ancuabe had flow cytometry to count CD4 cells on site but no facility had direct access to viral load monitoring. A fixed-dose combination of stavudine (d4T), lamivudine (3TC) and nevirapine (NVP) was the most prescribed ART regimen during the study period. In Lesotho, zidovudine (ZDV) or tenofovir (TDF) were used increasingly in first-line ART in recent years. Over $90 \%$ of patients were treated with a NVP-based regimen and only 3 different ART regimens were used to treat more than $95 \%$ of patients in Mozambique and Zimbabwe. In Lesotho over half of patients received an Efavirenz (EFV)-based initial regimen and the number of treatment options was higher due to the increasing availability of TDF. During the study period, protease inhibitors were not available or not used, depending on the site. 
A total of 9,271 patients started ART during the study period. We excluded patients with incomplete data on sex or age $(n=26)$, as well as patients who started ART less than 6 months before the closing date of the database $(n=1,520)$ : 7,725 patients were therefore included in analyses. In all clinics the majority of patients were women; overall 5,018 (65\%) were female (Table 2). Patients in Mozambique were younger than those starting ART in the two other countries. Median CD4 cell counts at start of ART ranged from 91 cells $/ \mu \mathrm{L}$ in Zaka, Zimbabwe, to 279 cells $/ \mu \mathrm{L}$ in Ancuabe, Mozambique. The proportion of patients in advanced clinical stage (WHO stage III/IV) was high in all clinics ranging from $48.3 \%$ in Seboche, Lesotho, to $94.8 \%$ in Chiure, Mozambique.

\section{Patients with no follow-up after starting ART}

Overall 449 patients (5.8\%) were not seen after the ART initiation visit, with large variations between countries (Table 1). In Mozambique, $21.8 \%$ of patients who started ART in Ancuabe and $8.9 \%$ of patients from Chiure did not return, whereas in Zimbabwe and Lesotho, the proportion of patients with no follow-up was below 5\%. Younger age and, to a lesser extent, male gender were associated with the probability of no follow-up (Table 3 ). Patients with CD 4 cell counts below 50 cells $/ \mu \mathrm{L}$ or missing CD 4 counts at baseline were also more likely not to return to the health facility after the start of ART. The risk of no follow-up was higher in Mozambique than in the two other countries. Finally, the probability of not returning after the first visit was higher in more recent years (Table 3).

\section{Mortality and LTFU}

Over 9,575 person-years of follow-up 1,319 patients (18.1\%) of the 7,276 patients with at least one follow-up visit were LTFU and 698 patients $(9.6 \%)$ died. The crude mortality rate was 7.3 (95\% CI 6.8-7.9) deaths per 100 person-years and the rate of LTFU 13.8 (13.114.5) per 100 person-years. There were important differences between countries: Figure 1 shows cumulative percentages from competing risk models of patients alive and on ART, patients lost to follow up and deaths for Zimbabwe, Lesotho and Mozambique. At 3 years, 9.0\% (95\% CI 7.6-10.5) were known to have died in Zimbabwe, 15.7\% (13.9-17.6) were lost to follow-up and 75.1\% (72.8-77.3) were alive and on ART. The figures were less favorable for Mozambique: $17.4 \%$ (14.9-20.0); 31.0\% (27.8-34.2) and 51.6\% (48.0-55.2), respectively, with Lesotho in an intermediate position (Figure 1).

Male gender, advanced clinical stage and CD4 cell counts below 50/ $\mu \mathrm{L}$ at ART initiation were consistently associated with higher mortality (Table 3). Older patients and females were less likely to be LTFU over the 3 years after starting ART, whereas the risk of LTFU was highest in patients without a CD4 cell count at the start of ART and if treatment was initiated after 2007, compared to the other groups. Patients in Zimbabwe were less likely to be lost to follow-up than those treated in Lesotho or Mozambique.

\section{DISCUSSION}

In this study of six ART programs in rural Mozambique, Zimbabwe and Lesotho, we found that the proportion of patients with no follow-up after starting ART differed from one setting to the other, reaching over $20 \%$ in the most remote setting in Mozambique. Retention in care over the first 3 years after the initiation of ART was unsatisfactory in all settings. Among patients followed up after the initial visit, mortality and loss to follow-up were particularly high in young men, and higher in Mozambique than in the other two countries.

The SolidarMed ART program serves poor, rural populations, in settings with little employment, low literacy and fragile, understaffed health systems. Indeed, one explanation for the poor retention is the limited capacity of the regional health systems, which were 
overwhelmed by the rapid scale-up of ART. ${ }^{8}$ This is illustrated by the two facilities in Mozambique: in Chiure, staff was carefully trained and pre-ART counseling consistently implemented. These efforts generally translated into stable treatment partnerships. In contrast, the delivery of ART in Ancuabe started later and grew faster, initially without external support, and the training of staff was limited. Pre-ART counseling was not standardized and often not performed, leading to the initiation of treatment in patients who were ill prepared to take on the challenges of life-long ART. Structural factors, as well as socio-cultural factors have previously been linked to poor outcomes in ART programs. ${ }^{17-19}$

Patients who do not come back to the health care facility after starting ART reflect a particular type of program failure. In our study over $5 \%$ of patients did not return after their first visit. Brinkhof et al. found somewhat lower rates of early loss in the African programs included in the ART-LINC collaboration; however, they mainly studied urban programs. ${ }^{8}$ Long-term retention was also low in our study, and particularly low in Lesotho and Mozambique where only $55 \%$ and $52 \%$ of patients, respectively, were alive and on ART at 3 years, fewer than the $72 \%$ estimated in the systematic review and meta-analysis of Fox et al. ${ }^{5}$ Again, most of the programs included in this review were from urban centers. A study of the barriers to accessing ART in Mozambique found that availability and cost of transport as well as clinic fees were major issues, particularly in rural areas, where the distance to the nearest clinic is large, public transport limited and unemployment high. ${ }^{20}$ In Lesotho, retention in care was below the estimates previously reported by Médecins sans Frontières in another setting in Lesotho. One explanation for this might be the large proportion of migrant workers to neighboring South Africa, a sub-population known to be at higher risk of LTFU than the general population. ${ }^{21,22}$

Patients lost to follow-up might reflect unaccounted self-referrals to other $\operatorname{sites}^{23}$, but this is unlikely in rural Mozambique and Zimbabwe, where ART is not widely available. In Lesotho, patients are routinely referred to peripheral health centers when clinically stable, but these patients will continue to be included in the common database. Men and patients under 30 years of age were more likely to be LTFU. Low retention in care of these groups is an important finding of our study and is consistent with other reports from Western, Eastern and Southern Africa. 7, 8, 24, 25 Because patients in this age group are more likely to have no follow-up, be LTFU or to die than older patients, they warrant special attention and support from ART programs, both in urban and rural settings. The increasing proportion of patients lost to follow-up in recent years must be of concern. Even though it is difficult to attribute these changes to specific factors, the increasing number of patients needing treatment in each ART program in the context of weak health systems with poor organizational and logistic capacities probably had a major impact on the retention in care of patients on ART. The levels of staffing were extremely low in many of these health care facilities, contributing to the high proportions of patients lost or without any follow-up after the initiation of ART.

In the presence of LTFU the mortality observed among patients remaining in care may substantially under-estimate mortality in all patients starting ART because mortality in patients LTFU tends to be high. Several methods have been used to address this issue, including statistical modeling based on mortality in patients traced in Kenya, and linkage of HIV cohort records with the national death registry in South Africa. ${ }^{26-29}$ A nomogram and web calculator (implemented at www.iedea-sa.org) has also been developed, based on the fact that mortality of all patients starting ART is a weighted average of mortality among patients LTFU and patients remaining in care. When entering plausible estimates of mortality in patients LTFU from a meta-analysis ${ }^{30}$ we found mortality at one year increased to about $11 \%$ (from 6\%) in Zimbabwe, to about 15\% (from 8\%) in Lesotho and to about $19 \%$ (from 12\%) in Mozambique. 
The lack of access to viral load monitoring and second-line ART may have contributed to the high mortality. When comparing outcomes of ART in South Africa, where viral load monitoring is routine, with Malawi and Zambia, where monitoring is based on CD4 cell counts, we recently found that at 3 years more patients were on a failing first-line regimen, fewer patients had switched to second-line ART and mortality was higher in Malawi and Zambia than in South Africa. ${ }^{31}$ These observational data are in contrast with the results from a randomized clinical trial ${ }^{32}$ and a mathematical modeling study ${ }^{33}$, which showed no or little association between viral load monitoring and mortality. As none of the health facilities included in the present study had access to viral load monitoring, it was not possible to assess the potential effect of different monitoring strategies on ART outcomes directly in our study. Finally, mortality was higher in men and in those with CD4 cell counts below $50 / \mu \mathrm{L}$ and advanced clinical stage, in line with the results of a prognostic model of patients starting ART in sub-Saharan Africa. ${ }^{34}$

There are several limitations to our study. First, the mortality we observed in patients remaining in care is bound to be an underestimate of mortality at the level of the program. Unfortunately, the limited resources mean that patients LTFU cannot be consistently traced by outreach teams. We could therefore not provide accurate information on program-level mortality, but estimated overall mortality based on published data on mortality in patients LTFU in sub-Saharan Africa ${ }^{26,27}$. We did not systematically compare outcomes of rural programs with those located in urban areas, but such an analysis is planned within the IeDEA collaboration. No information was available on causes of death. Laboratory equipment was sometimes out of order or could not be operated because of power breaks or lack of reagents, resulting in a considerable number of patients with missing CD4 counts at baseline. This was especially true for Mozambique: the programs either did not have a CD4 count device on site (Ancuabe) or faced frequent breakdowns of the device (Chiure). Severely ill and hospitalized patients were therefore started on ART without baseline CD4 count. Thus, the higher baseline CD4 counts in these 2 programs mainly relate to patients who were not hospitalized and in better health. Such selection bias was less important in Zimbabwe and Lesotho, where baseline CD4 count measurements seemed to be missing essentially at random.

In conclusion, our study illustrates some of the challenges rural ART programs face in subSaharan Africa. In particular, our results document the difficulties in retaining patients in care after they initiate ART and must raise concerns about poor long term clinical outcomes in settings with weak health systems and limited access to viral load monitoring and secondline ART regimens. Approaches to improve retention in care in such settings have been described recently, including reduction of the frequency of visits in patients who are stable, down referral of ART and the involvement of peer support groups. ${ }^{12,35,36} \mathrm{Such}$ interventions need to be tailored to local contexts and implemented alongside health systems strengthening initiatives 37 .

\section{Acknowledgments}

We thank all study participants as well as Svend Capol, Rudolf Fischer, Markus Frei, Clemens Truniger and Gabriel Waldegg for helpful discussions and support.

\section{References}

1. World Health Organization. Progress Report 2010. Geneva, Switzerland: World Health Organization; 2010. Towards Universal Access. Scaling Up Priority HIV/AIDS Interventions in the Health Sector.

2. Mills EJ, Nachega JB, Buchan I, et al. Adherence to antiretroviral therapy in sub-Saharan Africa and North America: a meta-analysis. JAMA. Aug 9; 2006 296(6):679-690. [PubMed: 16896111] 
3. Nachega JB, Mills EJ, Schechter M. Antiretroviral therapy adherence and retention in care in middle-income and low-income countries: current status of knowledge and research priorities. Curr Opin HIV AIDS. Jan; 2010 5(1):70-77. [PubMed: 20046150]

4. Rosen S, Fox MP, Gill CJ. Patient retention in antiretroviral therapy programs in sub-Saharan Africa: a systematic review. PLoS Med. Oct 16.2007 4(10):e298. [PubMed: 17941716]

5. Fox MP, Rosen S. Patient retention in antiretroviral therapy programs up to three years on treatment in sub-Saharan Africa, 2007-2009: systematic review. Trop Med Int Health. Jun; 2010 15( Suppl 1):1-15. [PubMed: 20586956]

6. Sanne IM, Westreich D, Macphail AP, Rubel D, Majuba P, Van Rie A. Long term outcomes of antiretroviral therapy in a large HIV/AIDS care clinic in urban South Africa: a prospective cohort study. J Int AIDS Soc. 2009; 12:38. [PubMed: 20017918]

7. Nglazi MD, Lawn SD, Kaplan R, et al. Changes in programmatic outcomes during 7 years of scaleup at a community-based antiretroviral treatment service in South Africa. J Acquir Immune Defic Syndr. Jan 1; 2011 56(1):e1-8. [PubMed: 21084996]

8. Brinkhof MW, Dabis F, Myer L, et al. Early loss of HIV-infected patients on potent antiretroviral therapy programmes in lower-income countries. Bull World Health Organ. 2008; 86(7):559-567. [PubMed: 18670668]

9. Braitstein P, Brinkhof MW, Dabis F, et al. Mortality of HIV-1-infected patients in the first year of antiretroviral therapy: comparison between low-income and high-income countries. Lancet. 2006; 367(9513):817-824. [PubMed: 16530575]

10. Tassie JM, Baijal P, Vitoria MA, Alisalad A, Crowley SP, Souteyrand Y. Trends in retention on antiretroviral therapy in national programs in low-income and middle-income countries. J Acquir Immune Defic Syndr. Aug 1; 2010 54(4):437-441. [PubMed: 20351559]

11. Egger M, Boulle A, Schechter M, Miotti P. Antiretroviral therapy in resource-poor settings: scaling up inequalities? Int J Epidemiol. June 1; 2005 34(3):509-512. [PubMed: 15941732]

12. Bedelu M, Ford N, Hilderbrand K, Reuter H. Implementing antiretroviral therapy in rural communities: the Lusikisiki model of decentralized HIV/AIDS care. J Infect Dis. Dec 1; 2007 196( Suppl 3):S464-468. [PubMed: 18181695]

13. HIV/AIDS. SolidarMed position paper. SolidarMed. 2010. Available at: http://www.solidarmed.ch

14. Egger M, Ekouevi DK, Williams C, et al. Cohort Profile: The international epidemiological databases to evaluate AIDS (IeDEA) in sub-Saharan Africa. International Journal of Epidemiology. May 18.2011

15. Gooley TA, Leisenring W, Crowley J, Storer BE. Estimation of failure probabilities in the presence of competing risks: new representations of old estimators. Stat Med. 1999; 18(6):695-706. [PubMed: 10204198]

16. Fine JP, Gray RJ. A proportional hazards model for the subdistribution of a competing risk. Journal of the American Statistical Association. 1999; 94(446):496-509.

17. Geng EH, Bangsberg DR, Musinguzi N, et al. Understanding reasons for and outcomes of patients lost to follow-up in antiretroviral therapy programs in Africa through a sampling-based approach. J Acquir Immune Defic Syndr. Mar 1; 2010 53(3):405-411. [PubMed: 19745753]

18. Miller CM, Ketlhapile M, Rybasack-Smith H, Rosen S. Why are antiretroviral treatment patients lost to follow-up? A qualitative study from South Africa. Trop Med Int Health. Jun; 2010 15( Suppl 1):48-54. [PubMed: 20586960]

19. Auld AF, Mbofana F, Shiraishi RW, et al. Four-year treatment outcomes of adult patients enrolled in Mozambique's rapidly expanding antiretroviral therapy program. PLoS One. 2011; 6(4):e18453. [PubMed: 21483703]

20. Posse M, Baltussen R. Barriers to access to antiretroviral treatment in Mozambique, as perceived by patients and health workers in urban and rural settings. AIDS Patient Care STDS. Oct; 2009 23(10):867-875. [PubMed: 19803678]

21. Cohen R, Lynch S, Bygrave H, et al. Antiretroviral treatment outcomes from a nurse-driven, community-supported HIV/AIDS treatment programme in rural Lesotho: observational cohort assessment at two years. J Int AIDS Soc. 2009; 12(1):23. [PubMed: 19814814] 
22. Bygrave H, Kranzer K, Hilderbrand K, et al. Trends in loss to follow-up among migrant workers on antiretroviral therapy in a community cohort in Lesotho. PLoS One. 2010; 5(10):e13198. [PubMed: 20976289]

23. Geng EH, Nash D, Kambugu A, et al. Retention in care among HIV-infected patients in resourcelimited settings: emerging insights and new directions. Curr HIV/AIDS Rep. Nov; 2010 7(4):234 244. [PubMed: 20820972]

24. Ochieng-Ooko V, Ochieng D, Sidle JE, et al. Influence of gender on loss to follow-up in a large HIV treatment programme in western Kenya. Bull World Health Organ. Sep 1; 2010 88(9):681688. [PubMed: 20865073]

25. Ekouevi DK, Balestre E, Ba-Gomis FO, et al. Low retention of HIV-infected patients on antiretroviral therapy in 11 clinical centres in West Africa. Trop Med Int Health. Jun; 2010 15( Suppl 1):34-42. [PubMed: 20586958]

26. Egger M, Spycher BD, Sidle J, et al. Correcting mortality for loss to follow-up: a nomogram applied to antiretroviral treatment programmes in sub-Saharan Africa. PLoS Med. 2011; 8(1):e1000390. [PubMed: 21267057]

27. Brinkhof MW, Spycher BD, Yiannoutsos C, et al. Adjusting mortality for loss to follow-up: analysis of five ART programmes in sub-Saharan Africa. PLoS One. 2010; 5(11):e14149. [PubMed: 21152392]

28. Geng EH, Emenyonu N, Bwana MB, Glidden DV, Martin JN. Sampling-based approach to determining outcomes of patients lost to follow-up in antiretroviral therapy scale-up programs in Africa. JAMA. Aug 6; 2008 300(5):506-507. [PubMed: 18677022]

29. Fox MP, Brennan A, Maskew M, MacPhail P, Sanne I. Using vital registration data to update mortality among patients lost to follow-up from ART programmes: evidence from the Themba Lethu Clinic, South Africa. Trop Med Int Health. 2010; 15(4):405-413. [PubMed: 20180931]

30. Brinkhof MW, Pujades-Rodriguez M, Egger M. Mortality of patients lost to follow-up in antiretroviral treatment programmes in resource-limited settings: systematic review and metaanalysis. PLoS One. 2009; 4(6):e5790. [PubMed: 19495419]

31. Keiser $\mathrm{O}$, Chi BH, Gsponer T, et al. Outcomes of antiretroviral treatment in programmes with and without routine viral load monitoring in southern Africa. AIDS. Sep 10; 2011 25(14):1761-1769. [PubMed: 21681057]

32. Jourdain, GN-G-HN.; Le Coeur, S.; Traisaithit, P.; Barbier, S.; Techapornroong, M., et al. PHPT-3: A Randomized Clinical Trial Comparing CD4 vs Viral Load ART Monitoring/Switching Strategies in Thailand. 18th Conference on Retroviruses and Opportunistic Infections; Boston. 2011.

33. Phillips AN, Pillay D, Miners AH, Bennett DE, Gilks CF, Lundgren JD. Outcomes from monitoring of patients on antiretroviral therapy in resource-limited settings with viral load, CD4 cell count, or clinical observation alone: a computer simulation model. Lancet. Apr 26; 2008 371(9622):1443-1451. [PubMed: 18440426]

34. May M, Boulle A, Phiri S, et al. Prognosis of patients with HIV-1 infection starting antiretroviral therapy in sub-Saharan Africa: a collaborative analysis of scale-up programmes. Lancet. Aug 7; 2010 376(9739):449-457. [PubMed: 20638120]

35. Decroo T, Panunzi I, das Dores C, et al. Lessons learned during down referral of antiretroviral treatment in Tete, Mozambique. J Int AIDS Soc. 2009; 12(1):6. [PubMed: 19419543]

36. Decroo T, Telfer B, Biot M, et al. Distribution of antiretroviral treatment through self-forming groups of patients in Tete province, Mozambique. J Acquir Immune Defic Syndr. Nov 13.2010

37. Savigny, Don de; Adam, Taghreed. Alliance for Health Policy and Systems Research. WHO; 2009. Systems thinking for health systems strengthening. 


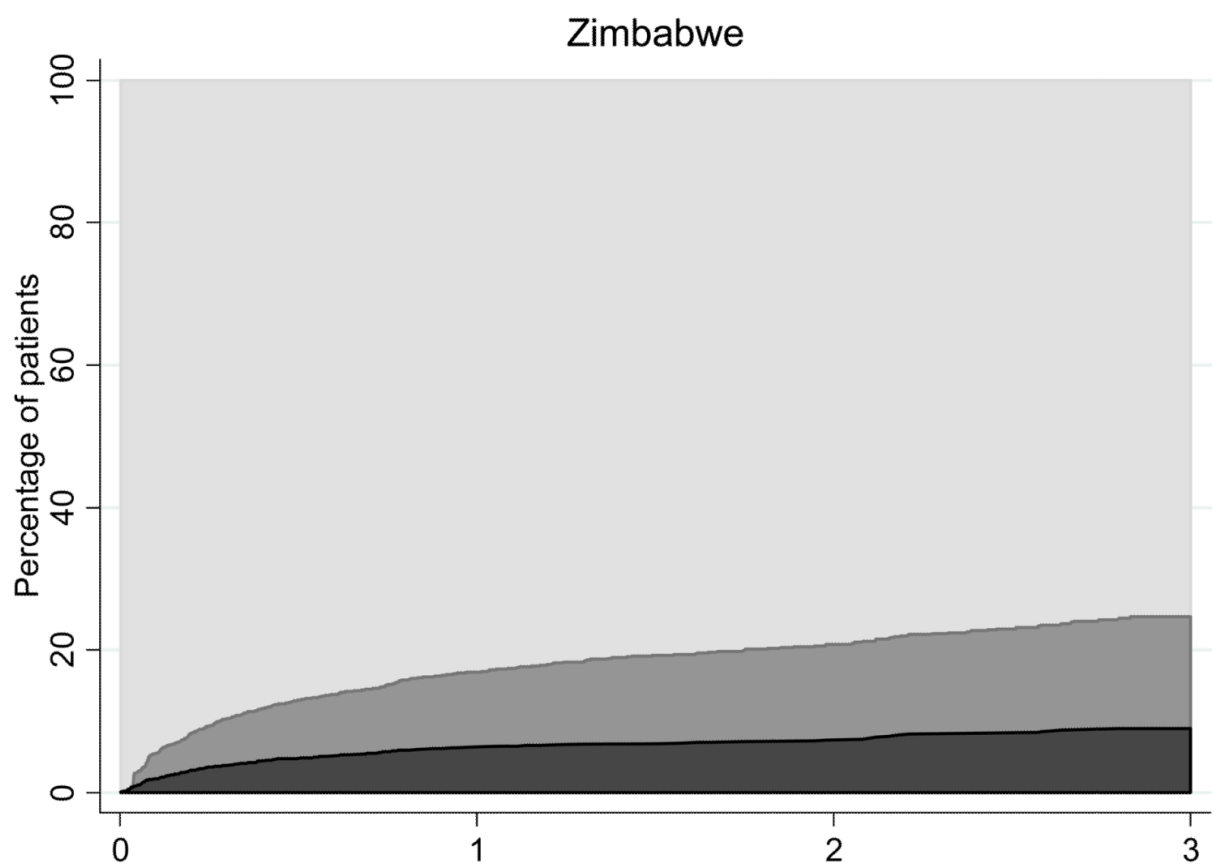

Competing risk estimates

\section{$(95 \% \mathrm{Cl})$}

Dead (\%)

$6.4(5.5-7.4)$

Lost to follow-up (\%)

$10.6(9.4-11.8)$

7.4 (6.4-8.5)

$9.0(7.6-10.5)$

Retained in program (\%)

$83.1(81.7-84.5)$

$79.2(77.5-80.8)$

$15.7(13.9-17.6)$ 


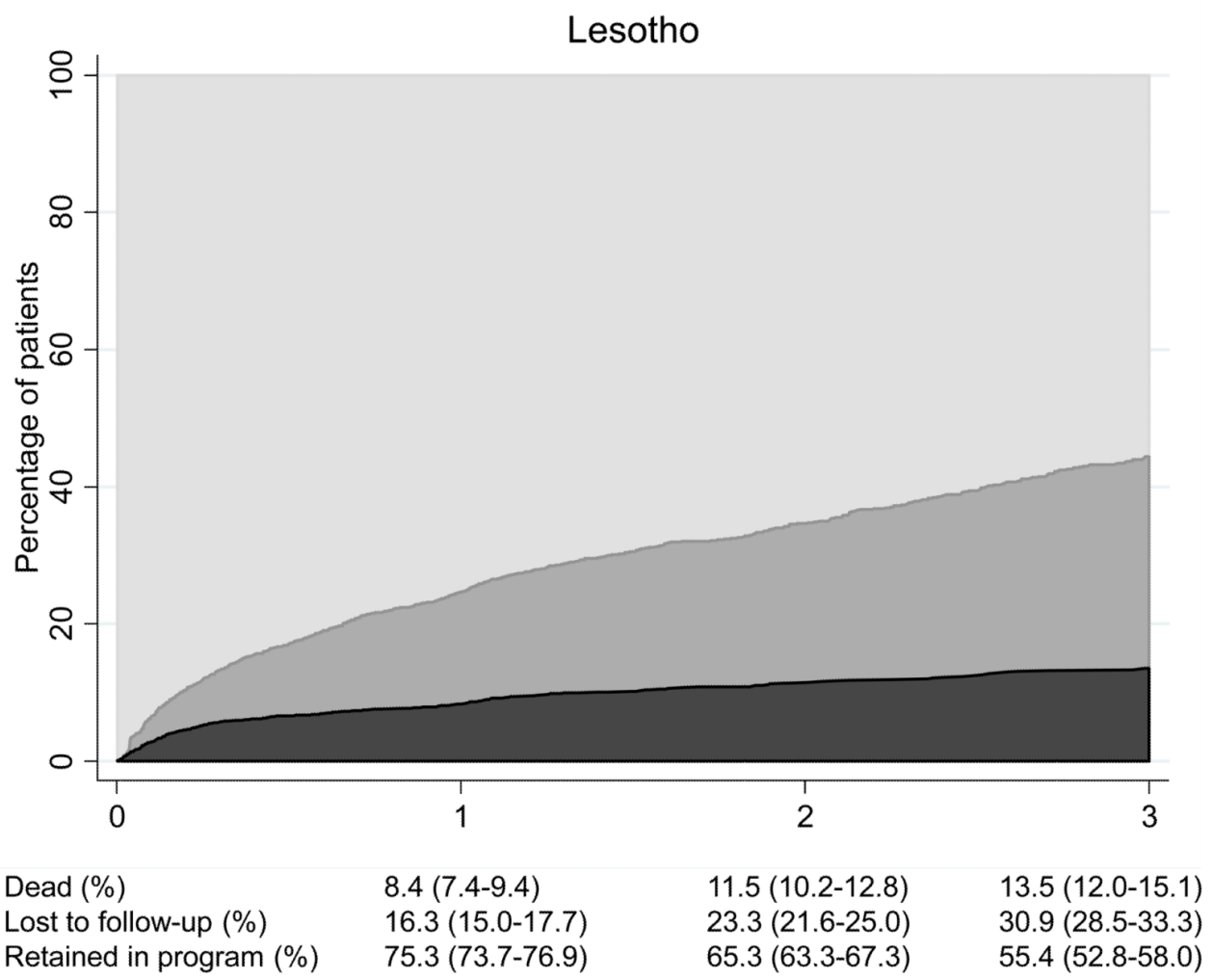




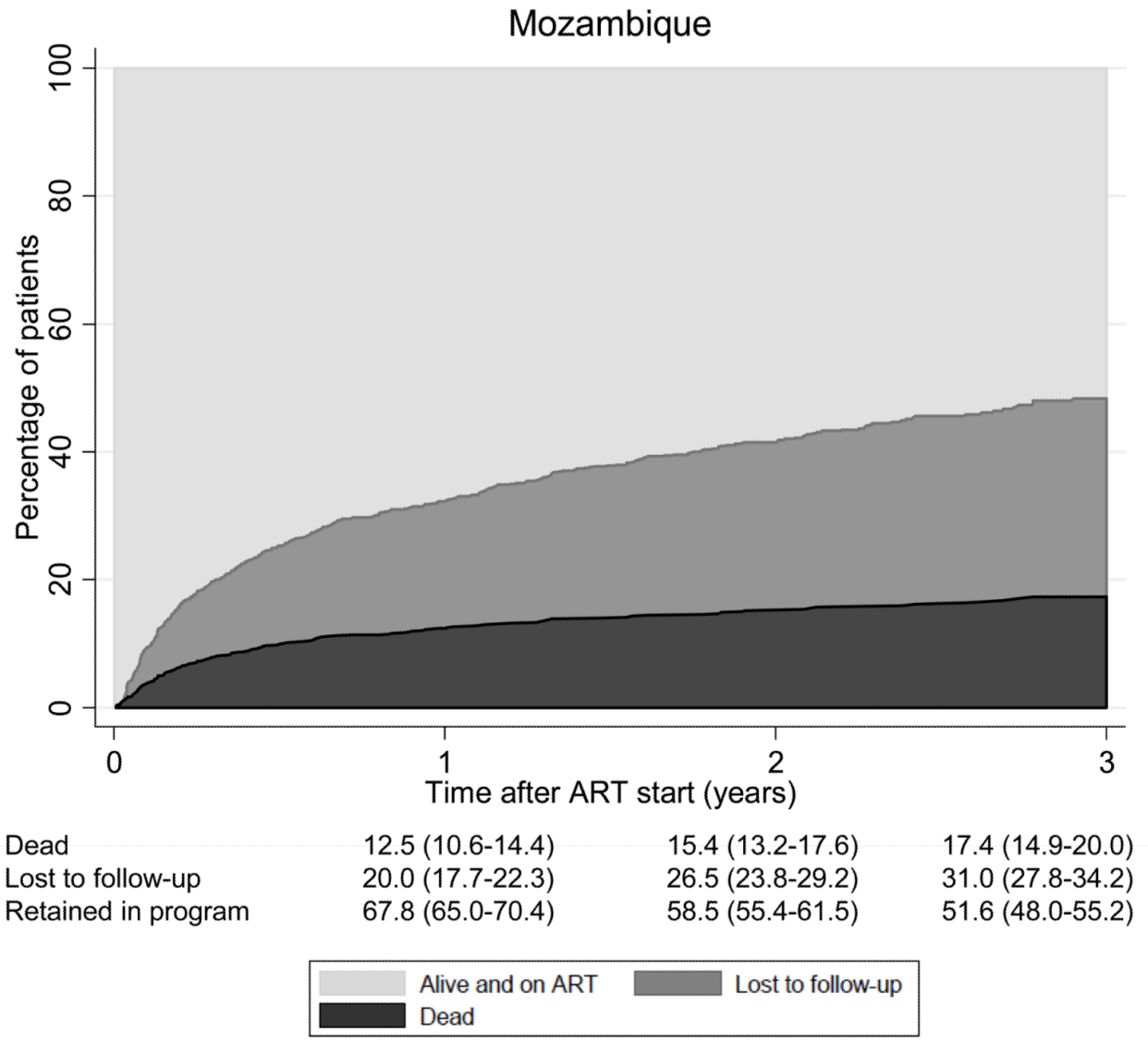

Figure 1. 


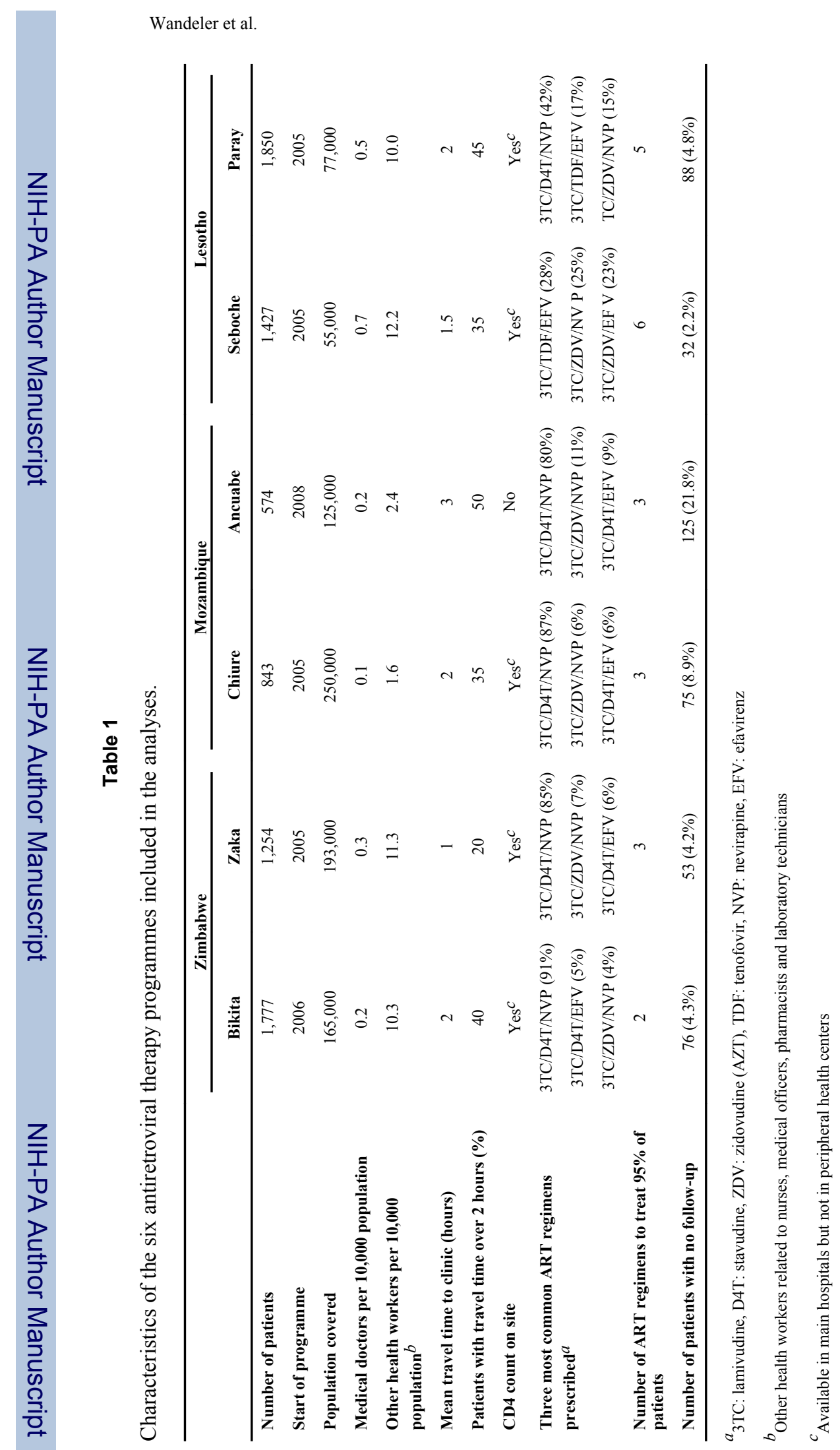




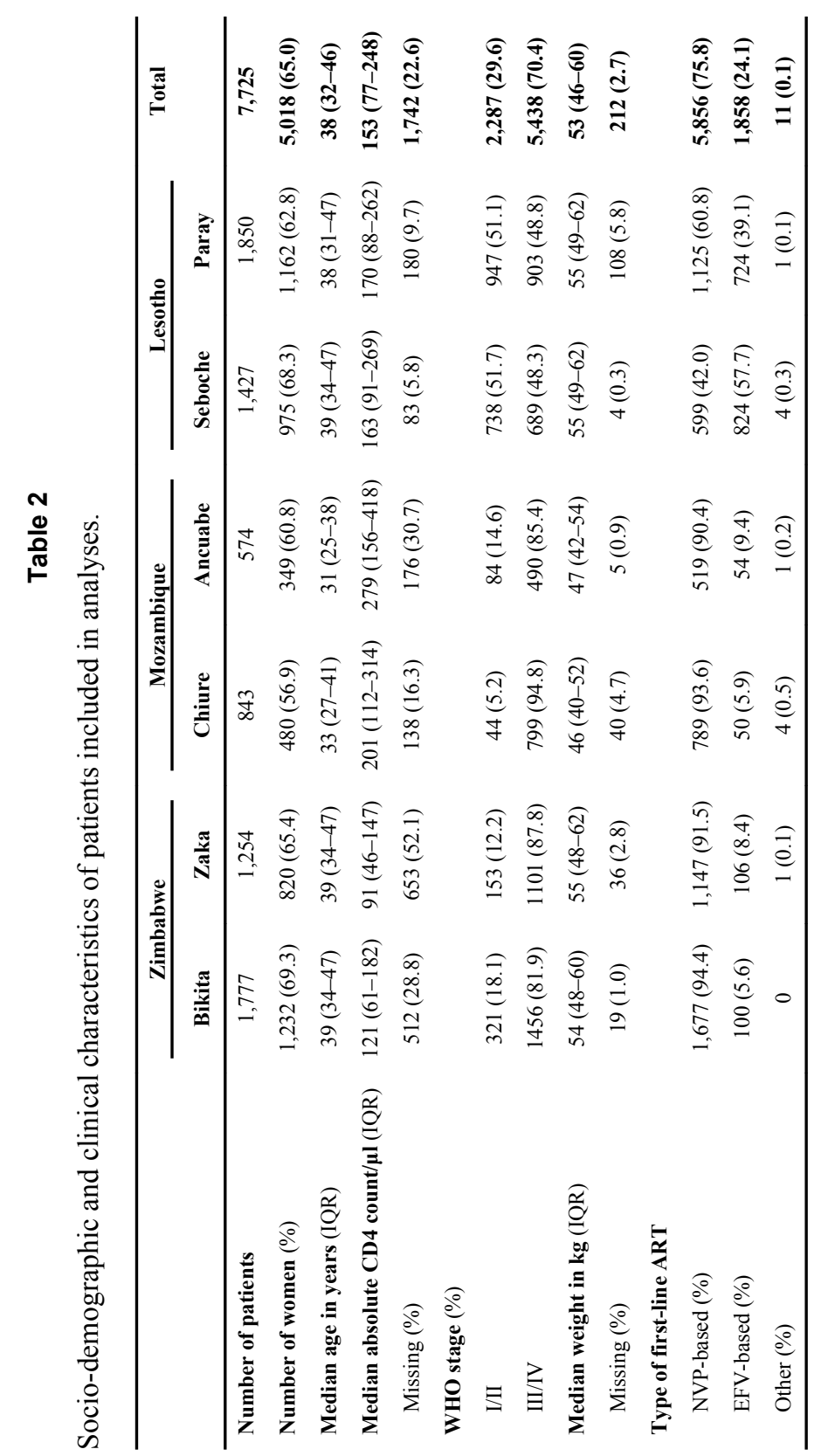




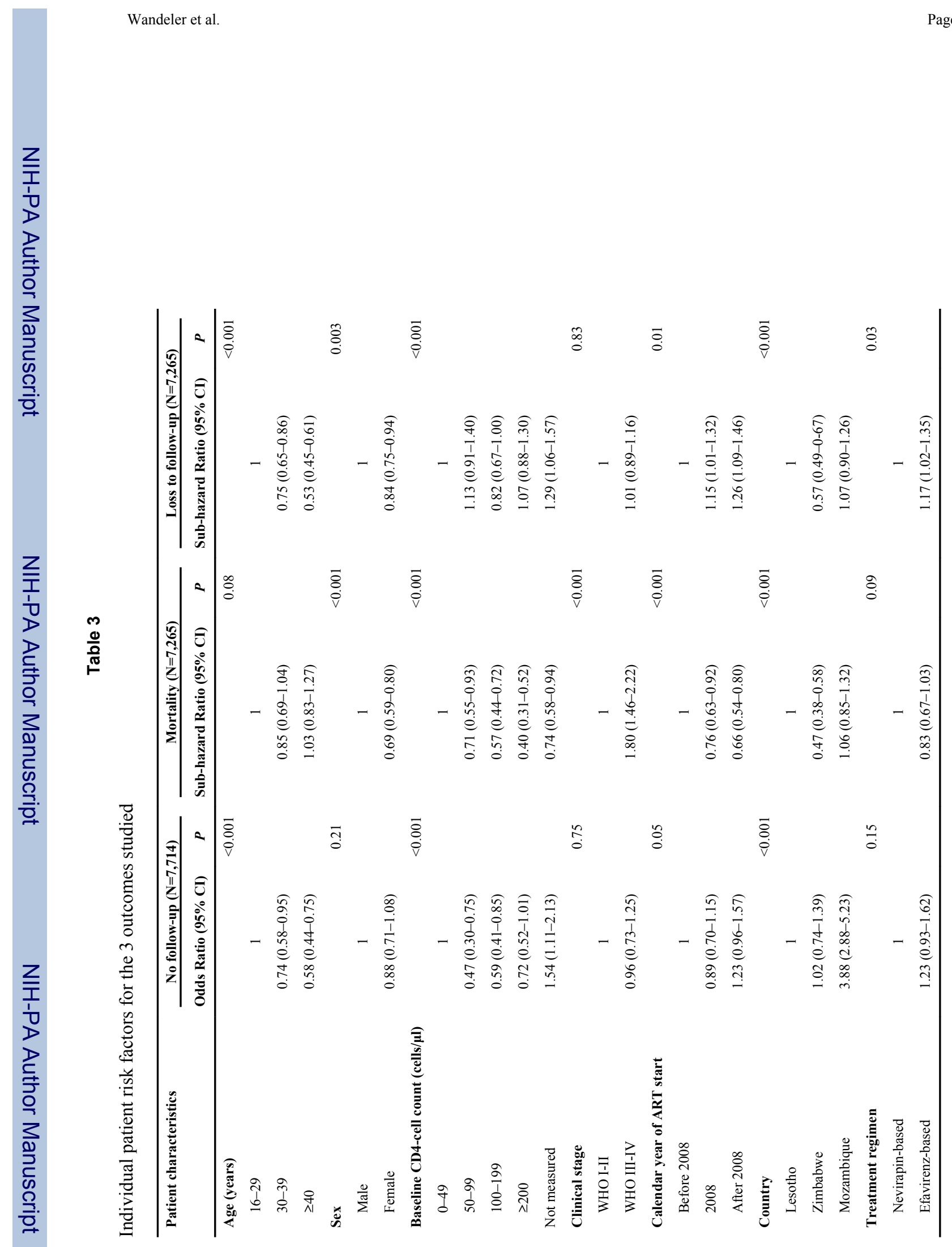


Wandeler et al.

Page 16

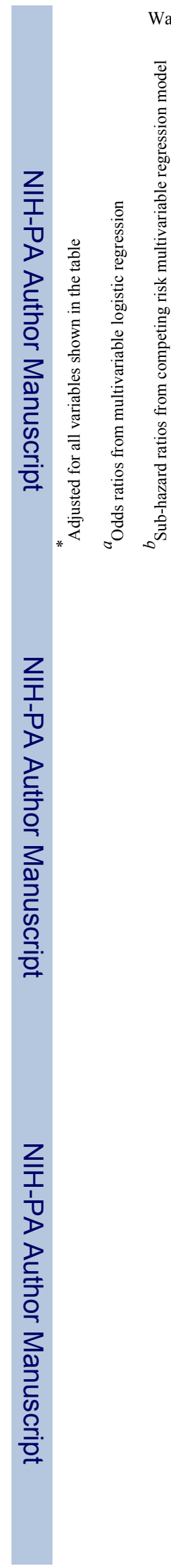

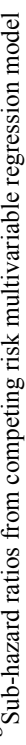

